\title{
PROCESSAMENTO E CARACTERIZAÇÃO DE BLENDAS DE POLIESTIRENO COM RETARDANTES À CHAMA
}

\author{
P.P. FURTAT, D. MACHADO, S.A.C ABARCA e R.A.F. MACHADO \\ Universidade Federal de Santa Catarina, Departamento de Engenharia Química e Engenharia de \\ Alimentos \\ E-mail para contato: plinario@gmail.com
}

\begin{abstract}
RESUMO - Blendas híbridas orgânico-inorgânicas contendo poliestireno (PS) e o precursor inorgânico poli(vinilsilazano) (HTTs), com os retardantes à chama trifenil fosfato (TFF), trifenilfosfano (TFFo) foram obtidos por mistura física direta em uma extrusora mono-rosca. $\mathrm{O}$ efeito retardante à chama foi caracterizado pela aplicação do teste vertical ASTM D-635 e o teste de inflamabilidade UL94 HB. A interação química dos aditivos na matriz polimérica foi analisada utilizando a espectroscopia de infravermelho por transformada de Fourier (FTIR) e microscopia eletrônica de varredura (MEV). As amostras apresentaram boa processabilidade e a adição do precursor alterou o aspecto do PS. Os resultados revelaram que a adição do precursor cerâmico e dos compostos organofosforados aumentaram a estabilidade térmica e a retardância à chama do PS significativamente, de acordo com a concentração de compostos adicionados nas blendas. $\mathrm{O}$ efeito sinérgico entre o retardante de fase gasosa trifenilfosfano e a formação de uma camada cerâmica causada pela adição de HTTs foi observada no produto final.
\end{abstract}

\section{INTRODUÇÃO}

O Poliestireno (PS) é um dos polímeros mais utilizados industrialmente, nas mais diferentes áreas e aplicações. Características como transparência, facilidade de coloração e processabilidade o tornam bastante útil em diversos setores industriais, como o da construção civil, o alimentício e a produção de componentes de peças eletro-eletrônicas (Borelly, 2002). Em seu estado puro, o poliestireno é altamente inflamável, ocorrendo a despolimerização e a liberação de grandes quantidades de substâncias e oligômeros facilmente inflamáveis como monômeros, dímeros e trímeros de estireno, hidrocarbonetos como benzeno e tolueno e ainda compostos aromáticos que contenham oxigênio (Svec et al., 1990).

Existem diversos métodos de se reduzir a inflamabilidade do poliestireno, descobertos através da pesquisa e aplicados com sucesso na indústria. $\mathrm{O}$ uso de compostos halogenados é uma das maneiras mais utilizadas na atualidade e de melhor resposta. Porém a utilização desses compostos tem sido gradualmente proibida, devido à liberação de gases tóxicos nocivos tanto ao meio ambiente como aos seres vivos. (Gallo; Agnelli, 1998). Por essa razão, o estudo de desenvolvimento de novos materiais com propriedades de retardância à chama tem crescido com o decorrer dos anos.

Recentemente, compostos contendo silício, incluindo carbonitretos de silício, conhecidos como precursores inorgânicos, estão sendo estudados como retardantes à chama na preparação de 
polímeros híbridos orgânico-inorgânicos. Esses materiais atuam como retardantes à chama de fase condensada. O mecanismo de retardância proposto por Fina, Tabuani e Camino (2010) para materiais carregados com precursores inorgânicos mostra que, em temperaturas próximas às de decomposição dos polímeros $\left(250-300{ }^{\circ} \mathrm{C}\right)$, esses compostos produzem camadas cerâmicas superficiais que impedem a transferência de calor da chama para o polímero e dificultam a difusão de oxigênio e compostos voláteis do material queimado para a chama, contribuindo para a extinção da frente de queima. Os Principais precursores inorgânicos estudados para esta finalidade são os polissilazanos (PSZ), polisiloxanos (PSS) e os polisilsesquixanos (POSS).

Existem poucos trabalhos na literatura técnico-científica que abordem a produção de compósitos de polímeros orgânicos e precursores inorgânicos com propriedades de retardância à chama. Abarca et al. (2013), estudaram a incorporação do precursor inorgânico polivinilssilazano pelos métodos de polimerização em massa e por solvente, alcançando um compósito com características hibridas das partes orgânica e inorgânica, como o aumento da estabilidade térmica e, consequentemente, da retardância à chama do material. Coan et al. (2012) avaliaram e compararam a estabilidade térmica e a proteção de um substrato de cobre revestido por compósitos híbridos de Poli(metil metacrilato), (PMMA) e polivinilssilazano e metais recobertos apenas por PMMA, aplicando, em ambos os casos, a técnica de dip coating. Os resultados mostraram um material $43 \%$ mais estável termicamente em comparação com o polímero puro, além de uma forte adesão do compósito ao substrato metálico.

A mistura de componentes no estado fundido é a técnica mais atrativa de incorporação de cargas inorgânicas em matrizes poliméricas, devido aos baixos custos, alta produtividade, compatibilidade com as técnicas de processamento convencionais de polímeros fundidos e é ecologicamente favorável uma vez que não é necessário utilizar solventes orgânicos voliteis (Cardoso, 2006). O procedimento consiste no aquecimento de uma mistura do polímero e das cargas inorgânicas, a uma temperatura superior ao ponto de fusão para polímeros semicristalinos, ou acima da Tg para os polímeros amorfos. Para esse processo, geralmente utiliza-se uma extrusora, que vai aquecer o polímero e as cargas, e com o movimento que a rosca do equipamento efetua, ocorre a mistura desses componentes.

Logo, o estudo do processamento de blendas híbridas pelos processos físicos, como injeção, extrusão e intercalação no estado fundido, é essencial para viabilizar a aplicação dessa nova classe de materiais no setor industrial, tornando-se uma alternativa ecológica e ambientalmente correta para o uso dos compostos halogenados. Neste trabalho será analisada a incorporação de precursores inorgânicos do grupo dos polissilazanos, juntamente com retardantes organofosforados, em uma matriz orgânica de PS cristal.

\section{MATERIAIS E MÉTODOS}

\subsection{Preparação das amostras}

Poliestireno (PS, N2650, Innova S.A, Brasil), Vinilpolissilazano (HTTs, Clariant, Alemanha), trifenilfosfato (TFF), trifenilfosfano (TFFo) fornecidos pela Sigma Aldrich (Alemanha), foram utilizados nessa pesquisa.

As blendas de PS, precursor inorgânico e aditivos foram processadas em uma extrusora mono rosca modelo 14LD30, marca AX Plásticos, no Laboratório de Controle de Processos do 
Departamento de Engenharia Química e Engenharia de Alimentos da UFSC. A extrusora possui três zonas de aquecimento, as quais após de vários testes foram definidas entre a faixa de temperatura de 147 a $159{ }^{\circ} \mathrm{C}$ e $42 \mathrm{rpm}$ da rosca de mistura. Após o processamento, as blendas foram trituradas e reprocessadas, a fim de se alcançar um material mais homogêneo.

\subsection{Caracterização térmica}

Para a análise da inflamabilidade das blendas poliméricas, foram utilizadas as normas internacionais ASTM D-635 horizontal e UL94. O teste horizontal compara a taxa relativa de queima e o tempo de queima de plásticos, moldados em placas, painéis ou chapas planas. $\mathrm{O}$ teste horizontal foi realizado em triplicata, e o vertical em quintuplicata, como requerem as respectivas normas.

\subsection{Caracterização estrutural}

A análise de Espectroscopia de Infravermelho por transformada de Fourier (FTIR) foi utilizada para descrever a possível interação das cargas na estrutura do polímero. Essas análises foram realizadas no Laboratório de Controle de Processos do Departamento de Engenharia Química da UFSC. O equipamento utilizado foi o FTIR marca Shimadzu, modelo IRPrestige 2, pelo método de confecção de pastilhas de brometo de potássio $(\mathrm{KBr})$.

Para a avaliação da morfologia das blendas obtidas por extrusão, amostras dos materiais foram analisadas por intermédio da Microscopia Eletrônica de Varredura (MEV). As análises foram conduzidas no Laboratório Central de Microscopia Eletrônica (LCME) da UFSC. Para isso foi utilizado um microscópio eletrônico de varredura, marca JEOL modelo JSM6400, operando com filamento de tungstênio de $15 \mathrm{kV}$.

\section{RESULTADOS E DISCUSSÃO}

\subsection{Processamento das amostras}

Várias amostras foram processadas, alterando-se a proporção em massa de cada um dos aditivos. Na tabela 1 têm-se as composições das amostras processadas na extrusora:

Tabela 1 - Composição das amostras preparadas

\begin{tabular}{|c|c|c|c|c|}
\hline Amostra & PS (\%) & HTTs(\%) & TFF(\%) & TFFo(\%) \\
\hline PS & 100 & 0 & 0 & 0 \\
\hline PSh04 & 90 & 9 & 0 & 1 \\
\hline PSh06 & 90 & 9 & 1 & 0 \\
\hline PSh08 & 90 & 9 & 0 & 0 \\
\hline PSh14 & 80 & 18 & 0 & 2 \\
\hline PSh16 & 80 & 18 & 2 & 0 \\
\hline PSh18 & 80 & 18 & 0 & 0 \\
\hline PSh24 & 80 & 10 & 0 & 10 \\
\hline PSh26 & 80 & 10 & 10 & 0 \\
\hline PSh28 & 80 & 10 & 0 & 0 \\
\hline
\end{tabular}


Todas as amostras apresentaram boa processabilidade, além de um aspecto uniforme. A adição de HTTs altera a cor da blenda, tornando-a opaca e esbranquiçada, e a adição de TFF deixa a blenda levemente amarelada, além de um aspecto áspero na superfície dos corpos de prova. Por outro lado, a adição de TFFo no PS não altera a cor das amostras. Na Figura.1 estão ilustrados o aspecto dos tipos de blendas processadas, onde tem-se o polímero puro na figura (a), e os sistemas PS/TFFo, PS/TFF, PS/HTTs, PS/HTTs/TFFo e PS/HTTs/TFF nas Figuras (b), (c), (d), (e) e (f), respectivamente.

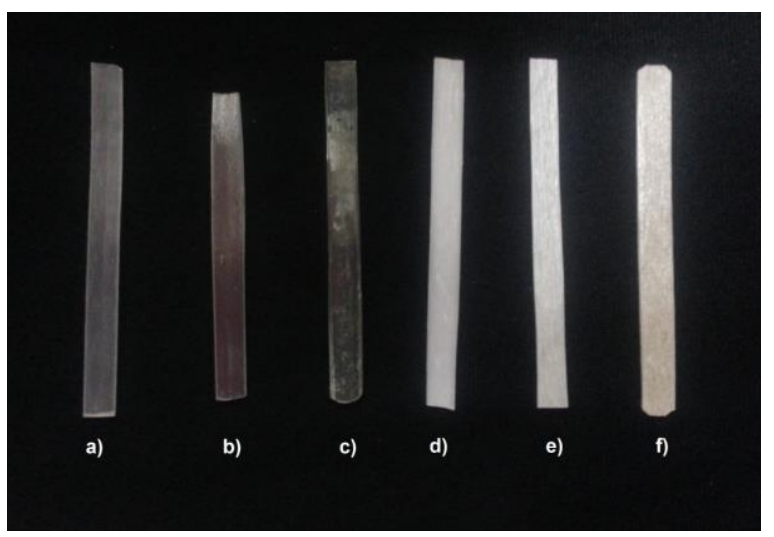

Figura 1 - Aspecto das blendas processadas na extrusora

\subsection{Caracterização térmica}

A aplicação do teste na amostra contendo apenas poliestireno apresentou um comportamento de queima análogo ao descrito pela literatura (YANG; SHIBASAKI, 1998), com corpo de prova queimando até o prendedor da amostra, e ocorrendo o gotejamento de material. Na tabela 2 encontra-se o resumo da aplicação da norma ASTM D-635 para as amostras. As blendas compostas por HTTs e TFFo apresentaram o melhor resultado no teste de inflamabilidade, com a chama se extinguido instantaneamente após a retirada fonte de calor. Na Figura 2 observa-se a amostra antes e depois da aplicação do teste.

Tabela 2 - Resultado do teste de inflamabilidade horizontal ASTM - D635

\begin{tabular}{|c|c|c|c|c|c|}
\hline Amostra & $\begin{array}{c}\text { Comprimento } \\
\text { queimado[mm] }\end{array}$ & $\begin{array}{c}\text { Tempo de } \\
\text { queima [s] }\end{array}$ & $\begin{array}{c}\mathrm{V} \\
{[\mathrm{mm} / \mathrm{min}]}\end{array}$ & Gotejamento & Classificação \\
\hline PSp & 75 & 51,04 & 88,24 & Sim & NC \\
\hline PSh04 & $*$ & $*$ & $*$ & Não & HB \\
\hline PSh06 & 28 & 41,86 & 40,13 & Sim & HB \\
\hline PSh08 & 75 & 83,17 & 54,22 & Sim & HB \\
\hline PSh14 & $*$ & $*$ & $*$ & Não & HB \\
\hline PSh16 & 4 & 5,57 & 21,54 & Sim & HB \\
\hline PSh18 & 16 & 32,21 & 29,8 & Sim & HB \\
\hline
\end{tabular}




\begin{tabular}{|c|c|c|c|l|l|} 
PSh24 & $*$ & $*$ & $*$ & Não & HB \\
\hline PSh26 & 2 & 4,63 & 38,88 & Sim & HB \\
\hline PSh28 & 10 & 17,63 & 34,03 & Sim & HB \\
& & & & & \\
\hline
\end{tabular}
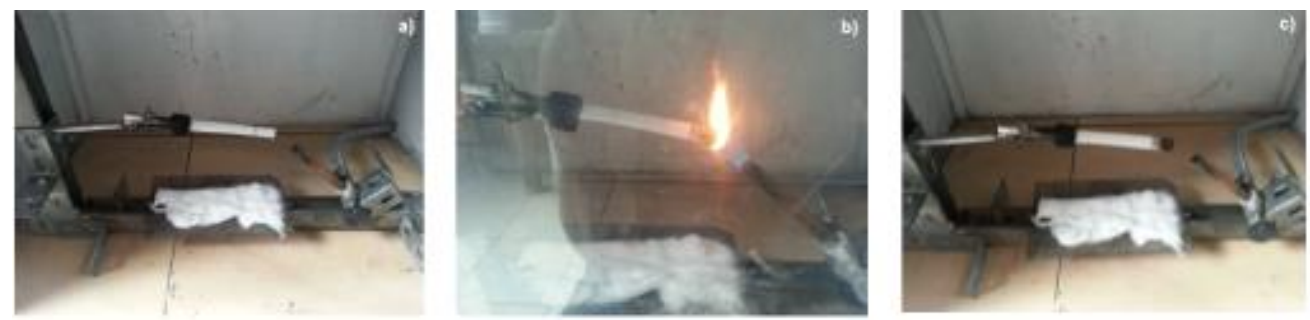

Figura 2 - Amostra PSh14 : a)antes , b) no momento e c) depois da aplicação da chama

As blendas compostas por HTTs e TFFo apresentaram o melhor resultado no teste de inflamabilidade, com a chama se extinguido instantaneamente após a retirada fonte de calor. Na Figura 2 observa-se a amostra antes e depois da aplicação do teste.

Já as amostras contendo HTTs e TFF apresentaram uma redução de aproximadamente $50 \%$ na taxa de queima, em relação ao PS puro, contudo, antes da queima cessar houve gotejamento de material. A amostra Psh16, que contém o dobro da quantidade de precursor inorgânico em relação a amostra PSh06, apresentou uma taxa linear de queima 46,3\% menor, demonstrando um aumento no retardo à chama pela criação de uma camada protetora que impede a queima do material.

Com isso pode-se observar que o aumento da quantidade de precursor inorgânico e retardante organofosforado adicionado na matriz polimérica diminui a taxa de queima do material, podendo agir de dois modos distintos, seja pela extinção da combustão em um tempo menor ou pelo retardo da propagação da chama na blenda. Para as amostras contendo PS/HTTs/TFFo, que não apresentaram gotejamento na aplicação da norma ASTM D-635, foi também aplicado o teste de inflamabilidade UL 94 vertical. Na Tabela 3 estão dispostos os resultados da aplicação do teste UL 94 vertical nas amostras analisadas.

Tabela 3 - Aplicação do teste UL 94 vertical no PS e suas blendas

\begin{tabular}{|c|c|c|c|c|c|c|}
\hline Amostra & $\begin{array}{c}\mathrm{t} 1 \\
(\mathrm{~s})\end{array}$ & $\begin{array}{c}\mathrm{t} 2 \\
(\mathrm{~s})\end{array}$ & $\begin{array}{c}\mathrm{t} 1+\mathrm{t} 2 \\
(\mathrm{~s})\end{array}$ & Gotejamento & $\begin{array}{c}\text { Queima } \\
\text { até o fim }\end{array}$ & Classificação \\
\hline PSp & 53 & - & - & Sim & Sim & NC \\
\hline PSh04 & 18,41 & 31,62 & 50,03 & Sim & Sim & NC \\
\hline PSh14 & 7,58 & 12,9 & 20,48 & Sim & Não & V-2 \\
\hline PSh24 & 4,52 & 8,33 & 12,85 & Não & Não & V-0 \\
\hline
\end{tabular}

$\mathrm{Na}$ aplicação para o PS puro, o corpo de prova queimou até o prendedor, o que isoladamente já o faz não receber classificação pelo teste. A amostra PSh14 obteve a classificação V-2 pois ocorreu a queda de material e por conseguinte, a queima da camada de algodão. Já a amostra 
PSh04 não foi classificável, pois o corpo de prova foi queimado por inteiro, fator esse que entra em desacordo com as classificações da referida norma. A amostra PSh24, que apresenta um percentual de TFFo de $10 \%$ em massa, foi a única a receber a classificação V-0, a melhor classificação possível pela realização do teste. A Figura 4.6 mostra os momentos anterior e imediatamente posterior à aplicação do teste na amostra PSh24.
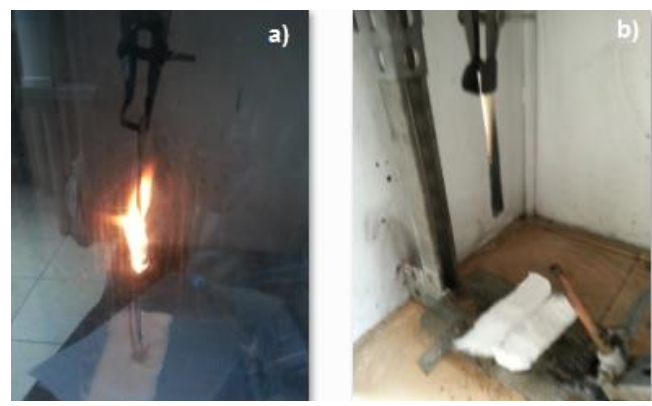

Figura 3 - Aplicação do teste de inflamabilidade de acordo com a norma UL 94 vertical

\subsection{Caracterização estrutural}

A Figura 4 relaciona o espectro do PS puro e as três amostras com a mesma razão de fases. Todos os espectros apresentam uma série de semelhanças decorrentes do fato de que são produzidos com a mesma fração do precursor inorgânico. Assim, os espectros apresentam picos associados principalmente com o polímero, entre as quais são ressaltados a presença de picos relativos à fase inorgânica. Nas blendas, notou-se a presença de picos relativos à fase inorgânica. Esses picos são mais evidentes nos comprimentos de 1260, 2164 e $3400\left[\mathrm{~cm}^{-1}\right]$, que caracterizam, respectivamente, a região de absorção da ligação $\mathrm{Si}-\mathrm{CH}_{3}$, ligação $\mathrm{Si}-\mathrm{H}$ e N-H.

Não foram observados picos relativos aos retardantes organofosforados. Uma razão possível é a sobreposição dos picos do PS, uma vez que os retardantes organofosforados possuem uma estrutura que contêm praticamente apenas um átomo de fósforo ligado a anéis aromáticos. A ausência de alterações dos picos do espectro de poliestireno mostra que a fase inorgânica se comporta apenas como um aditivo, não havendo interações significativas entre as fases. 


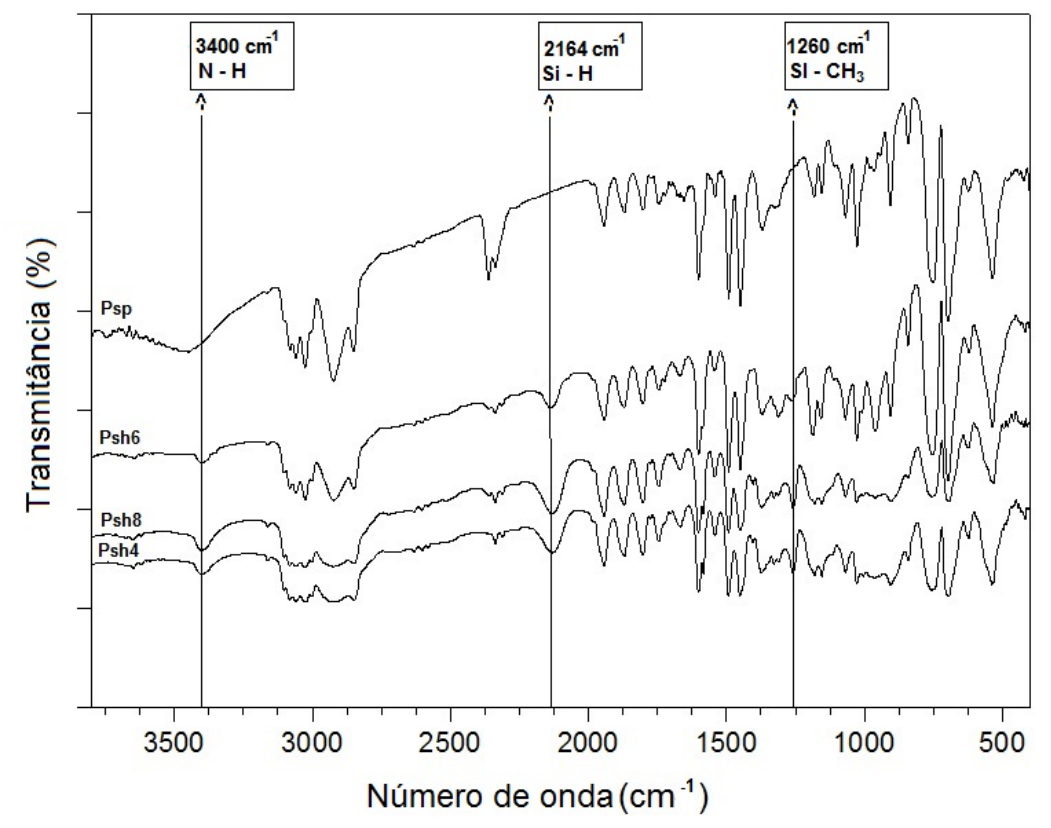

Figura 4 - análise de FTIR do PS e das blendas processadas

A microscopia eletrônica de varredura (MEV) foi utilizada com a finalidade de avaliar a morfologia das blendas obtidas pela extrusão do polímero juntamente com as cargas. A análise de MEV das amostras que apresentam o precursor inorgânico HTTs em sua composição comprova que, mesmo após a moagem e o reprocessamento das blendas, ainda existem regiões que apresentam uma alta concentração do mesmo, o que torna a as blendas heterogêneas. A figura 5 apresenta as imagens da estrutura do poliestireno puro (a) e das amostras PSh04, PSh06 e PSh08 (b,c e d, respectivamente). Essa fraca dispersão (ressaltada em vermelho) contribui para a diminuição da resistência das blendas, favorecendo o surgimento de regiões de aglomeração de precursor (mais resistentes) e regiões de concentração de polímero (mais frágeis).
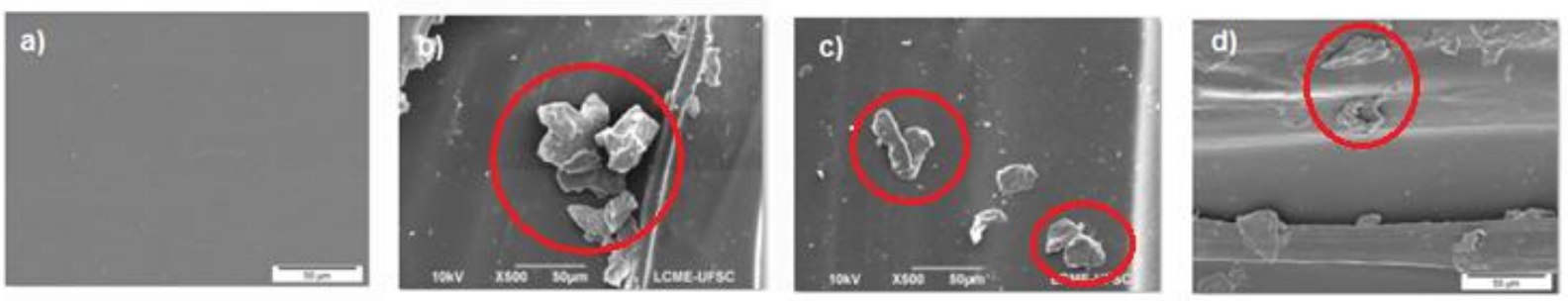

Figura 5 - Figura 4.18 - Microscopia eletrônica de varredura (MEV) das amostras.

\section{CONCLUSÕES}

A proposta deste trabalho esteve centrada no desenvolvimento de novas blendas híbridas de poliestireno juntamente com o precursor inorgânico vinilpolissilazano, e retardantes à chama 
organofosforados, com o intuito de se analisar o sinergismo entre esses compostos no aumento resistência à chama do novo material.

De forma geral, as blendas apresentaram uma boa processabilidade. Verificou-se que o HTTs atua na fase condensa da combustão do PS, criando uma camada cerâmica que protege a superfície do polímero, assim como os organofosforados atuam na fase gasosa, diminuindo a concentração de gases voláteis liberados pelo poliestireno.

Fica visível o sinergismo entre o precursor HTTs e o retardante à chama TFFo, uma vez que a adição de ambos no polímero evitou a ocorrência de gotejamento de material e causou a extinção instantânea da chama no corpo de prova, de acordo as normas aplicadas. Para o material alcançar a melhor classificação no teste de inflamabilidade UL94 vertical, foi necessário uma concentração de $10 \%$ de retardante e $10 \%$ de precursor na amostra.

Apesar dos bons resultados decorrentes das análises de inflamabilidade, a fase inorgânica não apresentou uma boa dispersão na matriz polimérica, criando regiões de aglomeração de HTTs. Soma-se a isso a diminuição da resistência mecânica da blenda, outra característica importante alterada pela adição do composto.

\section{REFERENCIAS}

ABARCA, S. A. C. et al. Synthesis and thermal characterization of silicon-based hybrid polymer. Chem. Eng. Trans, v. 32, p. 1621-1626, 2013.

BORELLY, D. F. Estudo comparativo da degradação de poliestireno e de poliestireno de alto impacto por envelhecimentos natural e artificial. Tese (Doutorado) - Universidade de São Paulo, 2002.

CARDOSO, L. P. Estudo da aplicação de hidróxidos duplos lamelares na remoção e liberação lenta de pesticidas. Tese (Doutorado) — Universidade de São Paulo (USP), 2006.

COAN, T. et al. Preparation of pmma/polysilazane coatings for metal surface protection. Em: Polymer Processing Society 28th Annual Meeting. [S.1.: s.n.], 2012.

FINA, A.; TABUANI, D.; CAMINO, G. Polypropylene/polysilsesquioxane blends. Eur. Pol. J, v. 46, n. 1 , p. $14-23,2010$.

GALLO, J. B.; AGNELLI, J. A. M. Aspectos do comportamento de polímeros em condições de incêndio. Pol., v. 8, p. 23 - 38, 1998.

SVEC, P. et al. Styrene-Based Plastics and Their Modification. New York, NY: Ellis Horwood, 1990. 277-299 p.

YANG, M.; SHIBASAKI, Y. Mechanisms of thermal degradation of polystyrene, polymethacrylonitrile, and their copolymers on flash pyrolysis. J. of Pol. v. 36, p. 2315-2330, 1998. 